\title{
Comparative Pathogenicity of Fusarium graminearum Isolates from Wheat Kernels in Korea
}

\author{
Sanghyun Shin ${ }^{1 \dagger}$, Jae-Han Son ${ }^{1}$, Jong-Chul Park ${ }^{1}$, Kyeong-Hoon Kim ${ }^{1}$, Young-mi Yoon ${ }^{1}$, Young-Keun \\ Cheong $^{1}$, Kyong-Ho Kim ${ }^{1}$, Jong-Nae Hyun ${ }^{1}$, Chul Soo Park ${ }^{2}$, Ruth Dill-Macky ${ }^{3}$, and Chon-Sik Kang ${ }^{1 *}$ \\ ${ }^{I}$ National Institute of Crop Science, RDA, Wanju 55365, Korea \\ ${ }^{2}$ Department of Crop Science and Biotechnology, Jeonbuk National University, Jeonju 54896, Korea \\ ${ }^{3}$ Department of Plant Pathology, University of Minnesota, 495 Borlaug Hall, 1991 Upper Buford Circle, St. Paul, MN \\ 55108, USA \\ †Present address: Animal and Plant Quarantine Agency, 167 Yongjeon-ro, Gimcheon 39660, Korea
}

(Received on February 1, 2018; Revised on May 5, 2018; Accepted on May 25, 2018)

Fusarium head blight (FHB) caused by Fusarium species is a major disease of wheat and barley around the world. FHB causes yield reductions and contamination of grains with trichothecene mycotoxins including; nivalenol (NIV), deoxynivalenol (DON), 3-acetyldeoxynivalenol (3-ADON), and 15-acetyldeoxynivalenol (15-ADON). The objectives of this study were to identify strains of $F$. graminearum isolated in Korea from 2012-harvested wheat grain and to test the pathogenicity of these NIV-and DON-producing isolates. Three hundred and four samples of wheat grain, harvested in 2012 in Chungnam, Chungbuk, Gyeongnam, Jeonbuk, Jeonnam, and Gangwon provinces were collected. We recovered 44 isolates from the 304 samples, based on the PCR amplification of internal transcribed spacer (ITS) rRNA region and sequencing. Our findings indicate that $F$. asiaticum was the predominant $(95 \%$ of all isolates) species in Korea. We recovered both $F$. asiaticum and $F$. graminearum from samples collected in Chungnam province. Of the 44 isolates recovered, 36 isolates had a NIV genotype while 8 isolates belonged to the DON genotype (3-ADON and 15-ADON). In order to characterize the pathogenicity of the strains

\footnotetext{
*Corresponding author.

Phone) +82-63-238-5225, FAX) +82-63-238-5205

E-mail) kcs1209@korea.kr

(c) This is an Open Access article distributed under the terms of the Creative Commons Attribution Non-Commercial License (http:// creativecommons.org/licenses/by-nc/4.0) which permits unrestricted noncommercial use, distribution, and reproduction in any medium, provided the original work is properly cited.
}

Articles can be freely viewed online at www.ppjonline.org. collected, disease severity was assessed visually on various greenhouse-grown wheat cultivars inoculated using both NIV-and DON-producing isolates. Our results suggest that Korean $F$. graminearum isolates from wheat belong to $F$. asiaticum producing NIV, and both $F$. graminearum and $F$. asiaticum are not significantly different on virulence in wheat cultivars.

Keywords : deoxynivalenol (DON), Fusarium graminearum, nivalenol (NIV)

Handling Associate Editor : Seo, Young-Su

Fusarium head blight (FHB), primarily caused by several Fusarium species Schwabe (teleomorph: Gibberella zeae), is a major disease of cereal crops around the world. The disease affects wheat, barley and other small grains and is especially prevalent in regions where hot and humid conditions prevail during the growing season (Parry et al., 1995). FHB significantly reduces yield through floret abortion and reductions in kernel size and weight (Bai and Shaner, 1994). Grain quality is also reduced due to physical damage to the kernel and as a result of the accumulation of mycotoxins including the trichothecene, deoxynivalenol (DON) and nivalenol (NIV), and the estrogenic mycotoxin zearalenone (Mirocha et al., 1989). The trichothecenes pose a significant risk to human and animal health because they inhibit eukaryotic protein synthesis and modify immune function (McMullen et al., 1997). In Korea, a severe epidemic of FHB on wheat and barley was reported in 1963 resulting in ca. $80-100 \%$ crop loss in the southern production area (Chun, 1963). Yield losses contributed to 
associated social problems resulting from both heavy economic losses and mycotoxicoses that developed in humans and farm animals from eating contaminated grain (Chung, 1975). More recently, serious yield losses have occurred in Korea in 1974, 1987, and 1998 (Ryu et al., 2011). DON have been reported as contaminants in wheat, barley, maize, and rice crops grown in Korea (Kim et al., 1993; Lee et al., 2009; Lee et al., 2012; Sohn et al., 1999).

FHB is primarily caused by $F$. graminearum, a species complex that is presently understood to consist of 16 phylogentically distinct lineages, based on a genealogical concordance phylogenetic species recognition (GCPSR) approach (Goswami and Kistler, 2005; Starkey et al., 2007; O'Donnell et al., 2000). Most lineages appear to be restricted to specific geographic regions. $F$. graminearum (lineage 7) is the dominant lineage most commonly associated with FHB worldwide, whereas $F$. asiaticum (members of lineage 6) is the major phylogenetic species associated with FHB in temperate regions of Asia (Goswami and Kistler, 2005). Lee et al. (2009) screened a large number of Fusarium isolates collected from rice in five provinces in Korea and reported that $F$. asiaticum was the predominate in all regions of Korea, although a small population of $F$. graminearum was found in eastern Korea.

The fungus $F$. graminearum produces C-8 keto trichothecenes mycotoxins including NIV and DON. Genetic studies have identified three genotypes, which are frequently referred to as 'chemotypes'. The three genotypes are: i) NIV chemotype which produces NIV and acetylated derivatives of NIV, ii) 3-ADON chemotype which produces primarily DON and 3-acetyldeoxynivalenol (3-ADON) and iii) $15-\mathrm{ADON}$ chemoype which produced primarily DON and 15-acetyldeoxynivalenol (15-ADON) (Miller et al., 1991; Mirocha et al., 1989). PCR-based assays have been developed for differentiating the NIV-, 3-ADON-, and 15-ADON-chemotypes among type B trichotheceneproducing species (Jennings et al., 2004; Lee et al., 2002). The three mycotoxin chemotypes recognized have different geographical distributions. The DON and NIV chemotypes have been reported in Africa, Asia, and Europe (Jennings et al., 2004; Miller et a., 1991; Shen et al., 2012; Suga et al., 2008). In Korea, the NIV chemotype has been identified as the predominant chemotype in the population of $F$. graminearum associated with wheat, barley, and rice while the DON chemotype has been most commonly reported in association with maize (Kim et al., 2003; Lee et al., 2001; Lee et al., 2009).

Isolates of $F$. graminearum vary in their ability to cause disease in different hosts (Akinsanmi et al., 2004; Carter et al., 2002; Goswami and Kistler, 2005). There are two important characteristics of $F$. graminearum; one is pathogenicity, which the ability to cause disease, and the other is aggressiveness, which may be measured as relative amount of disease that develops following inoculation of a host with a given isolate of the pathogen (Miedaner et al., 2008). The influence of mycotoxin chemotype in determining the pathogenicity of strains has been reported on in wheat and maize (Carter et al., 2002). However, most of studies on differences in pathogenicity and aggressiveness have been restricted to geographically localized collections of isolates. In wheat, Von der Ohe et al. (2010) reported that there were no significant differences between the mean FHB indices of isolates of the 3-ADON and 15-ADON chemotypes, although the average DON production by isolates of the 3-ADON chemotype was significantly higher than for isolates of the 15-ADON chemotype. In a greenhouse study, the isolates of the 3-ADON chemotype produced higher DON concentrations in inoculated wheat heads than the isolates of the 15-ADON chemotype, but no significant differences in aggressiveness were identified between isolates of the two chemotypes (Ward et al., 2008).

The objective of this study was to evaluate the mycotoxin production, pathogenicity and aggressiveness of Korean $F$. graminearum isolates, representing different mycotoxin chemotypes (NIV, DON, 3-ADON and 15-ADON), in wheat cultivars differing in their level of resistance or susceptibility.

\section{Materials and Methods}

Fusarium isolation. Wheat grain samples were collected from 17 counties in six provinces (Jeonbuk, Jeonnam, Chungbuk, Chungnam, Gangwon, and Gyeongnam) during June 2012. A total 304 samples were collected. For each sample ca. $500 \mathrm{~g}$ of grain was collected from the farm after harvest. Kernels (ca. 100 per collection) were surfaced sterilized in 3\% sodium hypochlorite for $2 \mathrm{~min}$, rinsed in sterile water for $2 \mathrm{~min}$ and placed on potato dextrose agar (PDA) amended with streptomycin $(50 \mathrm{mg} / \mathrm{l})$, in Petri plates. The plates were incubated for 4 to 5 days at $25^{\circ} \mathrm{C}$. All isolates recovered were transferred to PDA plates and subsequently single-spored. Single spore cultures were stored in $20 \%$ glycerol at $-80^{\circ} \mathrm{C}$. Isolate $\mathrm{GZ3639}(F$. graminearum) which is a DON-producing strain originating from Kansas USA (Desjardins et al., 1996) was included as a control.

Fungal genomic DNA extraction. Mycelia were harvested, from 5-7 day old cultures grown on PDA at $25^{\circ} \mathrm{C}$, with a sterile toothpick, transferred to a $1.5 \mathrm{ml}$ Eppendorf tube, frozen in liquid nitrogen, and ground to a fine powder. 
Genomic DNA was extracted using the Solg Genomic DNA Prep Kit (SolGent Co. Korea) according to the manufacturer's instructions. The concentration of total genomic DNA was estimated to be $5 \mathrm{ng} / \mu \mathrm{l}$ by comparison to DNA of known concentrations using agarose gel electrophoresis.

Identification of $\boldsymbol{F}$. graminearum species. Isolates were characterized by sequencing the internal transcribed spacer (ITS) region and translation elongation factor 1- $\alpha$ (TEF1) gene. The primer pair ITS1/ITS4 was used for polymerase chain reaction (PCR) amplification and the sequence was used to determine whether the isolates belonged to $F$. graminearum (Table 1). A PCR assay, with partial sequence of TEF1, was performed as described by O'Donnell (2000). PCR products were loaded on $1.5 \%$ agarose gel to check the amplification of $570 \mathrm{bp}$ and $700 \mathrm{bp}$ fragments for ITS and $T E F 1$, respectively. PCR products were purified using a QIAquick Gel Extraction Kit ${ }^{\circledR}$ (Qiagen; Valencia, CA). DNA sequencing was done by the Genotech Company, Korea. The ITS and TEF1 sequences obtained were aligned against the FUSARIUM-ID (http://isolate.fusariumdb.org/) and GenBank databases (http://www.ncbi.nlm.nih. gov).

Genotyping of Fusarium isolates for trichothecene production. Trichothecene chemotypes were determined by multiplex PCR based on Tri7 and Tri13 sequences (Lee et al., 2009; Zhang et al., 2010). The primer used in the Tril1 multiplex included N11/11R, 15D11/11R, and 3D11/11R that generated a $643 \mathrm{bp}$ fragment (indicating a NIV chemotype), a $424 \mathrm{bp}$ fragment (indicating a 15 -ADON chemotype), and a 342 bp fragment (indicating a 3-ADON chemotype), respectively (Table 1). For the PCRs, 50 ng of genomic DNA was used as the template in a $50 \mu \mathrm{l}$ reaction containing the PCR reagents ( $\mathrm{Li}$ et al., 2005). The PCRs were conducted in the thermal cycler (PTC-200, MJ Research) using the following conditions: $95^{\circ} \mathrm{C}$ for $5 \mathrm{~min}$; 30 cycles of $94^{\circ} \mathrm{C}$ for $40 \mathrm{~s}, 57^{\circ} \mathrm{C}$ for $40 \mathrm{~s}$ and $72^{\circ} \mathrm{C}$ for $1 \mathrm{~min}$; a final extension of $72^{\circ} \mathrm{C}$ for $5 \mathrm{~min}$. The PCR products were separated in a $1.5 \%$ agarose gel using electrophoresis. Gels were stained with ethidium bromide at $1 \mu \mathrm{g} / \mathrm{ml}$ and photographed under UV light.

Plant materials and growth condition. Twenty-eight winter wheat cultivars, all developed in Korea, were used in this study. The spring wheat cultivars Stoa, Tokai 66, Ning 7840, and Sumai 3 were also included as controls. Tokai 66, Ning 7840, and Sumai 3 are all highly resistant to FHB while Stoa is moderately susceptible (Waldron et al.,
1999). Seeds (4 per pot) were planted into Sunshine Mix \#1 (SunGro, Canada) in $15 \mathrm{~cm}$ diameter plastic pots. The plants were maintained in the greenhouse at the National Institute of Crop Science, at Iksan. The plants were maintained at $15-23^{\circ} \mathrm{C}$ during the day and $18-20^{\circ} \mathrm{C}$ at night.

Pathogenicity analyses. Four strains from each trichothecene chemotype were selected for the initial study. Four strains, JNW-1, CNW-3, CNW-6, and CNW-7, were selected for further analysis to assess the spread of the fungus and mycotoxin accumulation within wheat heads. The US strain GZ3639 (15-ADON chemotype) was included as a control. Two moderately resistant Korean winter wheat cultivars (Suan and Sukang) and three susceptible cultivars (Baekjong, Keumkang, and Jokyung) were selected for pathogenicity testing. Twenty plants (5 pots) were grown as described above. At anthesis, twelve heads per cultivar were inoculated by injecting $10 \mu \mathrm{l}$ of a conidial suspension $\left(1 \times 10^{5}\right.$ macroconidia/ml $)$ of a given isolate into a central floret of a spike. Inoculated heads were covered with plastic bags for 4 days to maintain the high humidity required for infection. Disease development was assessed using both disease severity and mycotoxin accumulation in harvested grain. Disease severity, the percentage of symptomatic spikelets per head, was assessed visually at 21 days after inoculation.

Mycotoxin analysis. Wheat spikelets were collected at maturity for analysis of trichothecenes (DON, 3-ADON, $15-\mathrm{ADON}$, and NIV). Mycotoxin concentrations in grain were determine using the seed bulked from 20 heads of each cultivar, since only limited amounts of grain were available. We observed that the seed set was greatly reduced and that the grain harvested from inoculated spikes was heavily damaged. The mycotoxin concentration of each grain sample was extracted and determined according to the method by Tanaka et al. (1985). Briefly, mycotoxins were extracted with acetonitrile/water (3:1), purified using Florisil columns and detected in a gas chromatograph-mass spectrometer (QP-5000: Shimadzu,Tokyo, Japan). The data are presented as mean values ( $\mu \mathrm{g} / \mathrm{g}$ dry wt. grain).

Statistical analysis. Statistical analysis were done using SAS software (SAS Institute, NC, USA) and using the Fisher's least significant difference (LSD) procedure. Correlations of FHB severity caused by $F$. graminearum isolate (CNW-6: DON chemotype) and $F$. asiaticum isolate (JNW-1: NIV chemotype) were estimated using SAS CORR procedure. 


\section{Results}

Isolation of Fusarium species from wheat kernels. Three hundred and four grain samples from the 2012 season were collected from Chungbuk, Chungnam, Gyeongnam, Jeonbuk, Jeonnam, and Gangwon provinces in Korea. From these samples 44 isolates were recovered using PDA media. The genomic DNA extracted from each isolate was used for the PCR amplification of the ITS rRNA region and the TEF1 gene (Table 1). Based on the ITS and TEF1 sequence alignment of the PCR product against the FUSARIUM-ID database, all 44 isolates were identified as
$F$. graminearum. These 44 isolates were used for trichothecene chemotyping.

Phylogenetic species identity and trichothecene chemotyping of $\boldsymbol{F}$. graminearum isolates. The species identity and trichothecene chemotype of the forty-four strains were investigated by the species-specific diagnostic PCR assay. The Tri13-specific primer pairs revealed 760 bp NIV-chemotype specific DNA fragments from 36 strains and 500$600 \mathrm{bp}$ DON-chemotype specific DNA fragments from the remaining eight strains (Fig. 1). F. asiaticum was clearly the dominant $(95 \%)$ species on wheat in Korea. Only two isolates, or $5 \%$ of the collected samples, were identified

Table 1. List of PCR primers and sequences used in this study along with their expected PCR product and reference

\begin{tabular}{clcc}
\hline Primer & \multicolumn{1}{c}{ Sequence (5'-3') } & PCR product & Reference \\
\hline ITS1 & TCCGTAGGTGAACCTGCG & $570 \mathrm{bp}$ & Park et al. (2010) \\
ITS4 & TCCTCCGCTTATTGATATGC & & \\
TEF-F & ATGGGTAAGGARGACAAGAC & $700 \mathrm{bp}$ & O'Donnell et al. (2000) \\
TEF-R & GGARGTACCAGSATCATGTT & & \\
GzTri13-p1 & AATACTA(A/C)AAG(C/T)CTAG(G/T)ACGACGC & $760 \mathrm{bp}$ & Lee et al. (2009) \\
GzTri13-p2 & GTG(A/G)T(A/G)TCCCAGGATCTGCGTGTC & $(\mathrm{NIV})$ & \\
N11 & CTTGTCAGGCGGCACAGTAG & $643 \mathrm{bp}$ & Zang et al. (2010) \\
$11 \mathrm{R}$ & TCAAAGGCCAGAGCAACCC & $(\mathrm{NIV})$ & \\
15D11 & AAGTATGGTCCAGTTGTCCGTATT & $424 \mathrm{bp}$ & Zang et al. (2010) \\
11R & TCAAAGGCCAGAGCAACCC & $(15-\mathrm{ADON})$ & \\
3D11 & GCAAGTCTGGCGAGGCC & $342 \mathrm{bp}$ & Zang et al. (2010) \\
11R & TCAAAGGCCAGAGCAACCC & $(3-\mathrm{ADON})$ & \\
\hline
\end{tabular}

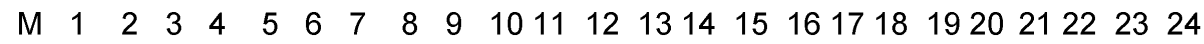

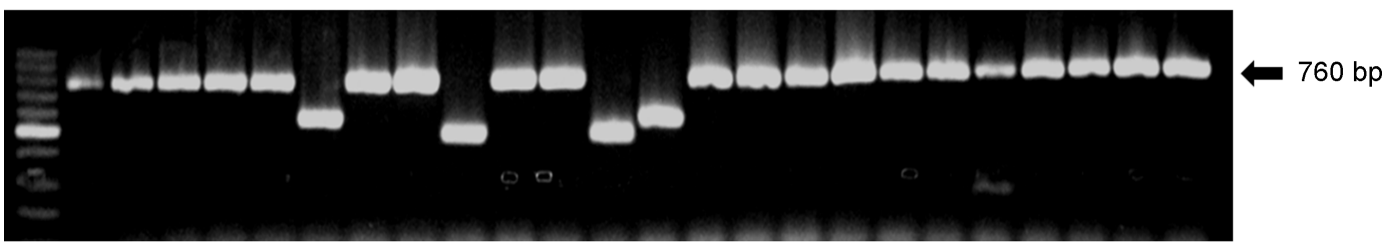

$\begin{array}{lllllllllllllllllllll}M & 25 & 26 & 27 & 28 & 29 & 30 & 31 & 32 & 33 & 34 & 35 & 36 & 37 & 38 & 39 & 40 & 41 & 42 & 43 & 44\end{array}$

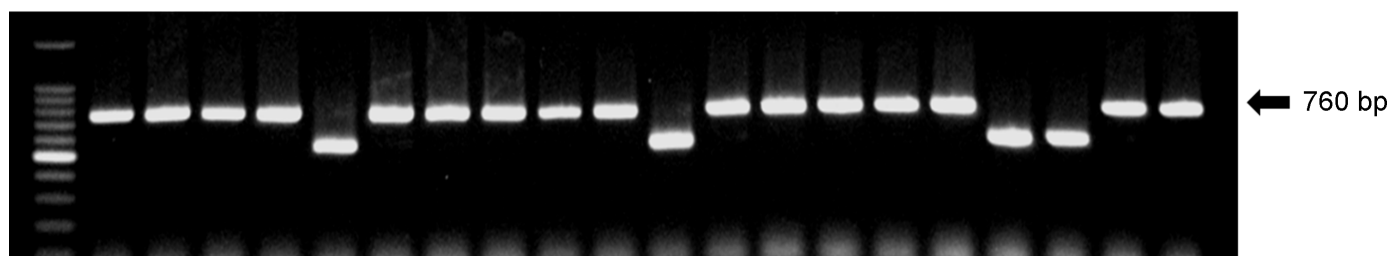

Fig. 1. PCR amplification patterns of the regions of Tri13 gene from DON- and NIV-producing F. graminearum genotypes. Lane M is the 100 bp DNA ladder. Lanes 6, 9, 12, 13, 29, 35, 41, and 42 have bands at 500-600 bp indicative of DON chemotypes, while the other lanes have bands at $760 \mathrm{bp}$ indicative of NIV chemotypes. 
Table 2. Geographical origin, species and trichothecene chemotypes of Fusarium graminearum clade isolates collected from different provinces in Korea

\begin{tabular}{|c|c|c|c|c|c|c|c|}
\hline \multirow{3}{*}{ Province } & \multirow{3}{*}{$\begin{array}{c}\text { No. of wheat } \\
\text { samples } \\
\text { collected }\end{array}$} & \multirow{3}{*}{$\begin{array}{c}\text { No. of } \\
\text { Fusarium strains } \\
\text { recovered }\end{array}$} & \multicolumn{3}{|c|}{ F. asiaticum } & \multicolumn{2}{|c|}{ F. graminearum } \\
\hline & & & \multicolumn{5}{|c|}{ Trichothecene chemotype } \\
\hline & & & NIV & $15-\mathrm{ADON}$ & 3-ADON & $15-\mathrm{ADON}$ & 3-ADON \\
\hline Jeonbuk & 53 & 5 & 5 & & & & \\
\hline Jeonnam & 98 & 1 & & & 1 & & \\
\hline Chungbuk & 14 & 2 & 2 & & & & \\
\hline Chungnam & 42 & 18 & 15 & & 1 & 1 & 1 \\
\hline Gangwon & 36 & 1 & 1 & & & & \\
\hline Gyeongnam & 61 & 17 & 13 & 4 & & & \\
\hline Total & 304 & 44 & 36 & 4 & 2 & 1 & 1 \\
\hline
\end{tabular}

as $F$. graminearum. We observed both $F$. asiaticum and $F$. graminearum in Chungnam province, but in all other provinces only $F$. asiaticum was found (Table 2). There were NIV, 3-ADON, and 15-ADON producers among the 44 isolates with the NIV genotype clearly dominant with 36 isolates recovered while only 6 isolates of the $F$. asiaticum had a DON chemotype (3-ADON and 15-ADON). Of the two isolates of $F$. graminearum recovered, there was one isolate each of the 3-ADON and 15-ADON chemotypes.

Pathogenicity of isolated on wheat. Four strains, representing the two chemotypes of $F$. graminearum (3-ADON and $15-\mathrm{ADON}$ ) and two of the three $F$. asiaticum chemotypes (NIV and 3-ADON) isolated, were selected for pathogenicity tests. The four strains differed in the extent of symptoms observed 21 days after inoculation on the five wheat cultivars (Table 3). The FHB severity of all the strains ranged from a minimum of $8 \%$ (on cultivar Sukang for isolate JNW-1) to a maximum of $66 \%$ (on cultivar Jokyung for isolate GZ3639). The GZ3639 isolate had highest severity on all five of the wheat genotypes inoculated in the study. There were no significant differences in the mean FHB severities, across the five wheat cultivars, between the NIV- and DON-producing isolates (Table 3).

The inoculated spikelets were harvested and used to determine the level of toxin accumulated following infection with the strains tested (Table 4). All strains produced detectable quantities of trichothecenes, though the amounts produced by individual strains varied. NIV was not detected in grain samples for all the isolates designated as NIV-chemotypes (data not shown). The range of DON produced by the strains was from below a detectable level to $21.0 \mathrm{ppm}$ (Table 4). Inoculation with the two 15ADON chemotypes resulted in the accumulation of higher amounts of DON in the FHB susceptible cultivar Jokyung.

Table 3. FHB severity of five wheat cultivars inoculated with three isolates of $F$. graminearum and two isolates of $F$. asiaticum 21 days after inoculation in a greenhouse test

\begin{tabular}{|c|c|c|c|c|c|c|c|}
\hline \multirow{2}{*}{ Isolate $^{1}$} & \multirow{2}{*}{ Chemotype } & \multicolumn{6}{|c|}{ FHB severity (\%) } \\
\hline & & Jokyung & Keumkang & Baekjong & Suan & Sukang & Mean $^{2}$ \\
\hline JNW-1 & NIV & 37.7 & 28.5 & 20.3 & 9.1 & 8.0 & $20.7^{\mathrm{a}}$ \\
\hline CNW-3 & 3-ADON & 57.2 & 31.1 & 20.4 & 10.1 & 9.7 & $25.7^{\mathrm{a}}$ \\
\hline CNW-6 & 15-ADON & 51.1 & 57.6 & 24.8 & 14.9 & 9.3 & $31.5^{\mathrm{a}}$ \\
\hline CNW-7 & 3-ADON & 43.1 & 30.4 & 24.7 & 11.2 & 10.2 & $23.9^{\mathrm{a}}$ \\
\hline GZ3639 & 15-ADON & 65.8 & 56.8 & 30.4 & 14.7 & 12.2 & $36.0^{\mathrm{a}}$ \\
\hline Mean $^{2}$ & & $51.0^{\mathrm{a}}$ & $40.9^{\mathrm{a}}$ & $24.1^{\mathrm{b}}$ & $12.0^{\mathrm{c}}$ & $9.9^{c}$ & \\
\hline
\end{tabular}

${ }^{1} \mathrm{JNW}-1$ is $F$. asiaticum (NIV chemotype), CNW-3 is F. graminearum (3-ADON chemotype), CNW-6 is $F$. graminearum (3-ADON chemotype), CNW-7 is F. asiaticum (3-ADON chemotype). Isolate GZ3639 is a F. graminearum (15-ADON chemotype) and served as the experimental control.

${ }^{2}$ Means within the column, or row, followed by different letters are significantly different at $\mathrm{p}=0.05$ according to Duncan's multiple range test. 
Table 4. Deoxynivalenol concentration of harvested grain from five wheat cultivars (Jokyung, Keumkang, Baekjong, Suan and Sukang) inoculated with isolates of F. graminearum (CNW-3, CNW-6 and GZ3639) and two isolates of F. asiaticum (JNW-1 and CNW-7) harvested 21 days after inoculation

\begin{tabular}{ccccccc}
\hline \multirow{2}{*}{ Isolate } & \multirow{2}{*}{$\begin{array}{c}\text { Isolate } \\
\text { chemotype }\end{array}$} & \multicolumn{5}{c}{ Deoxynivalenol $\left(\mathrm{mg} \mathrm{kg}^{-1}\right)$} \\
\cline { 3 - 7 } & & Jokyung & Keumkang & Baekjong & Suan & Sukang \\
\hline JNW-1 & NIV & ND $^{1}$ & ND & ND & ND & ND \\
CNW-3 & 3-ADON & 3.6 & 0.4 & ND & ND & ND \\
CNW-6 & 15-ADON & 8.9 & 5.7 & 1.2 & 3.7 & 3.2 \\
CNW-7 & 3-ADON & 15.9 & 4.2 & 1.3 & 0.7 & ND \\
GZ3639 & 15-ADON & 21.0 & 9.8 & 0.7 & 1.6 & 2.5 \\
\hline
\end{tabular}

${ }^{1} \mathrm{ND}$ : not detected

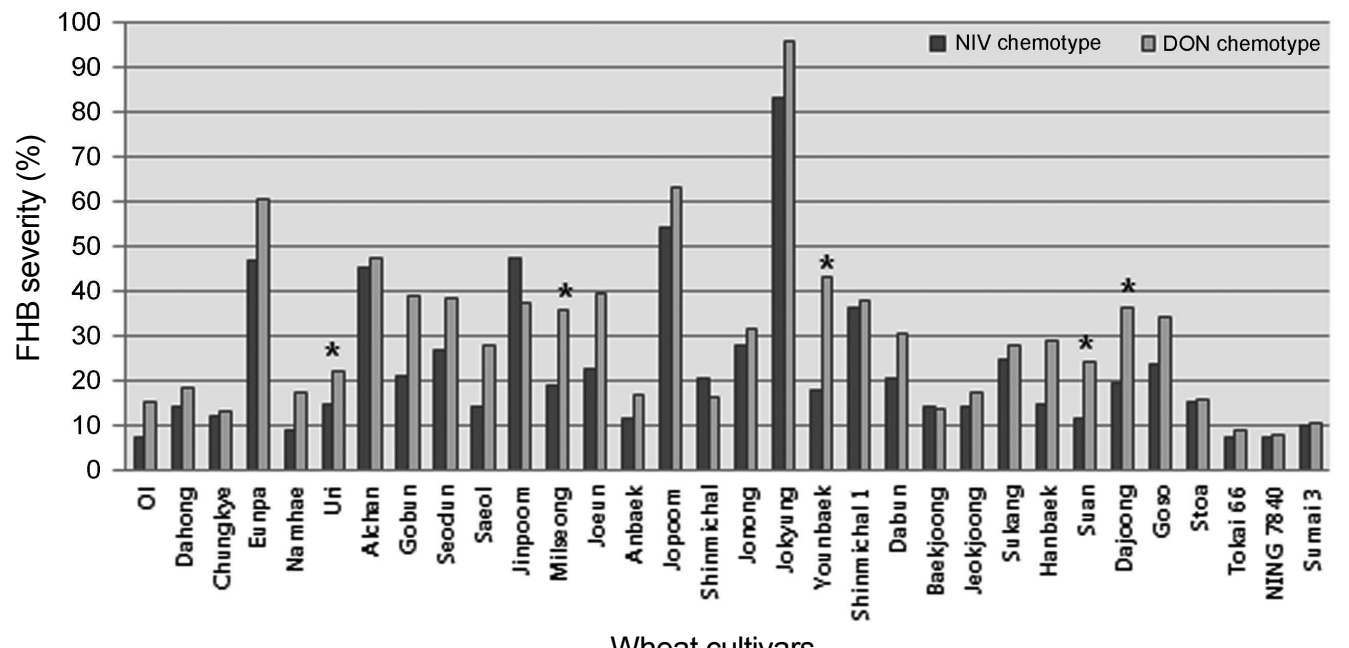

Wheat cultivars
Fig. 2. FHB severity for thirty-two wheat cultivars, inoculated with two $F u$ sarium isolates (CNW-6: a DON chemotype of $F$. graminearum and JNW-1: a NIV chemotype of $F$. asiaticum) in the greenhouse. Asterisks (*) indicate a significance difference, at the 0.05 level, between the FHB severities for the two isolates tested.
Twenty-eight Korean and five check wheat cultivars were evaluated for FHB severity with a strain representing each of the DON- and NIV-chemotypes (Fig. 2). There was a significant correlation between DON-chemotypes and NIV-chemotypes to disease development in the wheat head

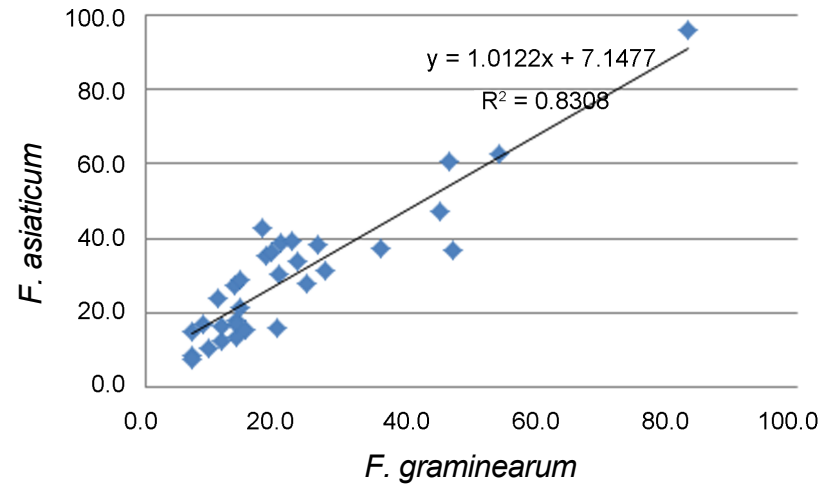

Fig. 3. Correlation of the Fusarium head blight severity caused by a $F$. graminearum isolate CNW-6 (DON chemotype) and $F$. asiaticum, isolate JNW-1 (NIV chemotype) on 32 wheat cultivars.
(Fig. 3). FHB severity was generally greater for the DON producing isolate tested than for the NIV producing isolated tested.

\section{Discussion}

In Korea, a severe epidemic of FHB on wheat and barley occurred in 1963, causing crop loss of $80-100 \%$ and the resulted in significant social problems (Chun, 1963). More recently, increases of FHB in wheat have been observed and may be the result of climate change. Conditions that favor FHB development may be more prevalent as a longer rainy season means that wheat is more likely to flower under conditions that favor FHB development. In this study, we identified forty-four F. graminearum isolates from 304 grain samples collected in 6 provinces, representing almost all the wheat growing areas Korea where FHB is recognized as a disease of importance. This report is the first evaluation of mycotoxin production and agressiveness for $F$. graminearum isolates representing the different myco- 
toxin chemotypes (NIV, DON, 3-ADON, and 15-ADON) found in association with wheat in Korea.

It has been proposed that the $F$. graminearum composition is dependent on geographic location and host. In the United States, Europe, and Canada, F. graminearum is predominate though it is also found in other parts of the world. In contrast, $F$. asiaticum is mainly restricted to Asia (Goswami and Kistler, 2005; O’Donnell et al., 2000; Starkey et al., 2007). F. asiaticum appears to have a southern distribution in China and Japan, while $F$. graminearum appears to be more commonly isolated from wheat and barley in the northern parts of these countries (Shen et al., 2012; Suga et al., 2008; Zhang et al., 2010; Zhang et al., 2013). Similarly in Korea, $F$. graminearum has been reported to be most common in the northern provinces, where most maize is produced, while $F$. asiaticum has been reported from the southern provinces where the majority of the rice and barley is produced (Lee et al., 2009). In our study, $F$. asiaticum was the predominant recovered in our sampling area which represented the southern provinces. Only $5 \%$ of the isolates we recovered from this southern region of Korea were $F$. graminearum (Table 2 ).

The relative proportions of $F$. asiaticum and $F$. graminearum in the population in a given region may depend on climate, rather than host. Qu et al. (2008) reported that $F$. graminearum was mainly distributed in the cooler regions of China, with the vast majority of $F$. asiaticum isolates were collected from warmer regions. In Japan, $F$. graminearum was found predominantly in the north, especially in the Hokkaido area, while $F$. asiaticum was found more frequently in southern regions (Suga et al., 2008). We found that $F$. graminearum was in the cooler western regions of Korea while $F$. asiaticum was more widely distributed across the warmer southern regions that were surveyed (Table 2). Consistent with our results, the population of Korean Fusarium isolates collected from rice also show that $F$. asiaticum dominates the warmer southern regions while $F$. graminearum dominates in cooler eastern rice production region of the country (Lee et al., 2009).

The capacity of strains within the $F$. graminearum complex to spread and cause disease on wheat is more likely to be the result of strain-specific, rather than lineage-specific, characteristics (Goswami and Kistler, 2005). Several studies have suggested that aggressiveness of $F$. graminearum may be correlated with toxin chemotypes. Ward et al. (2008) reported that $F$. graminearum isolates from the 3-ADON chemotype population in Canada produced significantly more trichothecenes (deoxynivalenol, 3-ADON and 15-ADON) overall and had significantly higher fecundity and growth rates in comparison to isolates from the 15-
ADON population. Gilbert et al. (2010) reported that there were no significant differences in aggressiveness between 3-ADON and 15-ADON chemotypes in Canada, although the 3-ADON isolate they tested produced significantly higher DON levels than the 15-ADON isolate following point inoculation in wheat in a greenhouse study. Our studies also showed that there was no significant difference in aggressiveness between isolates, representing the 3-ADON and $15-\mathrm{ADON}$ chemotypes, in their ability to cause FHB symptoms on wheat. Isolates of the 3-ADON and 15ADON chemotyope produce higher levels of DON in susceptible cultivars than on the moderately resistant cultivars tested (Table 4).

In this study aggressiveness was observed to be an isolate-specific, rather than a lineage-specific characteristic, although only a limited number of isolates were examined. There were no significant differences in the FHB severities between the two isolates except in the cultivars Uri, Milseong, Younbaek, Suan, and Dajoong (Fig. 2). In these five cultivars, the NIV-producing isolate generated significantly less disease than the DON-type isolate. These results suggest that the DON-producing isolate are generally more aggressive on wheat. DON and NIV have both been shown to act as virulence factors on wheat (Proctor et al., 2002). Carter et al. (2002) found no difference in the aggressiveness, towards wheat, of DON and NIV chemotypes of $F$. asiatiucm, although the NIV chemotypes were significantly more aggressive than the DON chemotypes towards maize. Gale et al. (2011) reported that DON producing strains of $F$. graminearum did accumulate four times more toxin, and spread significantly faster in a wheat head, than the NIV producing isolates tested in the United States. It is probable that aggressiveness is determined by a number of factors other than chemotype, but trichothecene production capacity may play a role in relative competitive ability on particular host species (Qu et al., 2008).

Our study records that both $15-\mathrm{ADON}$ and 3-ADON chemotypes of $F$. graminearum are found the northern part of Korea, although $F$. asiaticum is the predominant species associated with wheat in the population. There were no significant differences between NIV- and DON-producing isolates with respect to the relative level of resistance for the Korean wheat cultivars tested in this study.

\section{Acknowledgment}

This work was carried out with the support of Cooperative Research Program for Agriculture Science and Technology Development (Project No. PJ012464012018), Rural Development Administration, Republic of Korea. 


\section{References}

Akinsanmi, O. A., Mitter, V., Simpfendorfer, S., Backhouse, D. and Chakraborty, S. 2004. Identity and pathogenicity of Fusarium spp. isolated from wheat fields in Queensland and northern New South Wales. Aust. J. Agric. Res. 55:97-107.

Bai, G. and Shaner, G. 1994. Scab of wheat: prospects for control. Plant Dis. 78:760-766.

Carter, J. P., Rezanoor, H. N., Holden, D., Desjardins, A. E., Plattner, R. D. and Nicholson, P. 2002. Variation in pathogenicity associated with the genetic diversity of Fusarium graminearum. Eur. J. Plant Pathol. 108:573-583.

Chun, J. H. 1963. Epidemiological survey of human mycotoxicosis caused by scabby cereals. In: Research report on wheat and barley scab., ed. by Republic of Korea, pp. 385-507. Ministry of Agriculture and Forestry, Seoul, Korea (in Korean).

Chung, H. S. 1975. Cereal scab causing mycotoxicoses in Korea and present status of mycotoxin research. Kor. J. Mycol. 3:3136.

Desjardins, A. E., Proctor, R. H., Bai, G., McCormick, S. P., Shaner, G., Buechley, G. and Hohn, T. M. 1996. Reduced virulence of Trichothecene-nonproducing mutants of Gibberella zeae in wheat field tests. Mol. Plant-Microbe Interact. 9:775781.

Gale, L. R., Harrison, S. A., Ward, T. J., O’Donnell, K., Milus, E. A., Gale, S. W. and Kistler, H. C. 2011. Nivalenol-type populations of Fusarium graminearum and F. asiaticum are prevalent on wheat in southern Louisiana. Phytopathology 101:124-134.

Gilbert, J., Clear, R. M., Ward, T. J., Gaba, D., Tekauz, A., Turkington, T. K., Woods, S. M., Nowicki, T. and O'Donnell, K. 2010. Relative aggressiveness and production of 3- or 15-acetyl deoxynivalenol and deoxynivalenol by Fusarium graminearum in spring wheat. Can. J. Plant Pathol. 32:146152.

Goswami, R. S. and Kistler, H. C. 2005. Pathogenicity and in planta mycotoxin accumulation among members of the $\mathrm{Fu}$ sarium graminearum species complex on wheat and rice. Phytopathology 95:1397-1404.

Jennings, P., Coates, M. E., Walsh, K., Turner, J. A. and Nicholson, P. 2004. Determination of deoxynivalenol- and nivalenol-producing chemotypes of Fusarium graminearum isolated from wheat crops in England and Wales. Plant Pathol. 53:643-652.

Kim, J. C., Kang, H. J., Lee, D. H., Lee, Y. W. and Yoshizawa, T. 1993. Natural occurrence of Fusarium mycotoxins (trichothecenes and zearalenone) in barley and corn in Korea. Appl. Environ. Microbiol. 59:3798-3802.

Kim, H. S., Lee, T., Dawlatana, M., Yun, S. H. and Lee, Y. W. 2003 Polymorphism of trichothecene biosynthesis genes in deoxynivalenol- and nivalenol-producing Fusarium graminearum isolates. Mycol. Res. 107:190-197.
Lee, J., Chang, I. Y., Kim, H., Yun, S. H., Leslie, J. F. and Lee, Y. W. 2009. Genetic diversity and fitness of Fusarium graminearum populations from rice in Korea. Appl. Environ. Microbiol. 75:3289-3295.

Lee, J., Kim, H., Jeon, J. J., Kim, H. S., Zeller, K. A., Carter, L. L. A., Leslie, J. F. and Lee, Y. W. 2012. Population Structure of and Mycotoxin Production by Fusarium graminearum from Maize in South Korea. Appl. Environ. Microbiol. 78:21612167.

Lee, T., Oh, D. W., Kim, H. S., Lee, J., Kim, Y. H., Yun, S. H. and Lee, Y. W. 2001. Identification of deoxynivalenol- and nivalenol-producing chemotypes of Gibberella zeae. App. Environ. Microbiol. 67:2966-2972.

Lee, T., Han, Y. K., Kim, K. H., Yun, S. H. and Lee, Y. W. 2002. Tri13 and Tri7 determine deoxynivalenol- and nivalenolproducing chemotypes of Gibberella zeae. Appl. Environ. Microbiol. 68:2148-2154.

Li, H. P., Wu, A. B., Zhao, C. S., Scholten, O., Löffler, H. and Liao, Y. C. 2005. Development of a generic PCR detection of deoxynivalenol- and nivalenol-chemotypes of Fusarium graminearum. FEMS Microbiol. Lett. 243:505-511.

McMullen, M., Jones, R. and Gellenberg, D. 1997. Scab of wheat and barley: a re-emerging disease of devastating impact. Plant Dis. 81:1340-1348.

Miedaner, T., Cumagun, C. J. R. and Chakraborty, S. 2008. Population genetics of three important head blight pathogens Fusarium graminearum, F. pseudograminearum and F. culmorum. J. Phytopathol. 156:129-139.

Miller, J. D., Greenhalgh, R., Wang, Y. Z. and Lu, M. 1991. Trichothecene chemotypes of three Fusarium species. Mycologia 83:121-130.

Mirocha, C. J., Abbas, H. K., Windels, C. E. and Xie, W. 1989. Variation in deoxynivalenol, 15-acetyldeoxynivalenol, 3 -acetyldeoxynivalenol, and zearalenone production by Fusarium graminearum isolates. Appl. Environ. Microbiol. 55:1315-1316.

O’Donnell, K., Kistler, H. C., Tacke, B. K. and Caspar, H. H. 2000. Gene genealogies reveal global phylogeographic structure and reproductive isolation among lineages of Fusarium graminearum, the fungus causing wheat scab. Proc. Natl. Acad. Sci. U.S.A. 97:7905-7910.

Parry, D. W., Jenkinson, P. and McLeod, L. 1995. Fusarium ear blight (scab) in small grain cereals - a review. Plant Pathol. 44:207-238.

Park, Y., Shin, H. B., Kim, C. K., Roh, K. H., Yum, J. H., Yong, D., Jeong, S. H. and Lee, K. 2010. Identification of bacterial and fungal isolates by sequence analysis of 16S rRNA and internal transcribed spacer. Korean J. Clin. Microbiol. 13:34-39.

Proctor, R. H., Desjardins, A. E., McCormick, S. P., Plattner, R. D., Alexander, N. J. and Brown, D. W. 2002. Genetic analysis of the role of trichothecene and fumonisin mycotoxins in the virulence of Fusarium. Eur. J. Plant Pathol. 108:691-698.

Qu, B., Li, H. P., Zhang, J. B., Huang, T., Carter, J., Liao, Y. C. and Nicholson, P. 2008. Comparison of genetic diversity and 
pathogenicity of fusarium head blight pathogens from China and Europe by SSCP and seedling assays on wheat. Plant Pathol. 57:642-651.

Ryu, J. G., Lee, S., Lee, S. H., Son, S. W., Nam, Y. J., Kim, M., Lee, T. and Yun, J. C. 2011. Natural occurrence of Fusarium head blight and its mycotoxins in 2010-harvested barley and wheat grains in Korea. Res. Plant Dis. 17:272-279 (in Korean).

Shen, C. M., Hu, Y. C., Sun, H. Y., Li, W., Guo, J. H. and Chen, H. G. 2012. Geographic distribution of trichothecene chemotypes of the Fusarium graminearum species complex in major winter wheat production areas of China. Plant Dis. 96:1172-1178.

Sohn, H. B., Seo, J. A. and Lee, Y. W. 1999. Co-occurrence of Fusarium mycotoxins in mouldy and healthy corn from Korea. Food Addit. Contam. 16:153-158.

Starkey, D. E., Ward, T. J., Aoki, T., Gale, L. R., Kistler, H. C., Geiser, D. M., Suga, H., Tóth, B., Varga, J. and O’Donnell, K. 2007. Global molecular surveillance reveals novel Fusarium head blight species and trichothecene toxin diversity. Fungal Genet. Biol. 44:1191-1204.

Suga, H., Karugia, G. W., Ward, T., Gale, L. R., Tomimura, K., Nakajima, T., Miyasaka, A., Koizumi, S., Kageyama, K. and Hyakumachi, M. 2008. Molecular characterization of the Fusarium graminearum species complex in Japan. Phytopathology. 98:159-166.

Tanaka, T., Hasegawa, A., Matsuki, Y., Ishii, K. and Ueno, Y. 1985. Improved methodology for the simultaneous detection of the trichothecene mycotoxins deoxynivalenol and nivalenol in cereals. Food Addit. Contam. 2:125-137.

Von der Ohe, C., Gauthier, V., Tamburic-Ilincic, L., Brule-Babel, A., Fernando, W. G. D., Clear, R., Ward, T. J. and Miedaner, T. 2010. A comparison of aggressiveness and deoxynivalenol production between Canadian Fusarium graminearum isolates with 3-acetyl and 15-acetyl deoxynivalenol chemotypes in field-grown spring wheat. Eur. J. Plant Pathol. 127:407417.

Waldron, B. L., Moreno-Sevilla, B., Anderson, J. A., Stack, R. W. and Frohberg, R. C. 1999. RFLP mapping of a QTL for Fusarium head blight resistance in wheat. Crop Sci. 39:805-811.

Ward, T. J., Clear, R. M., Rooney, A. P., O’Donnell, K., Gaba, D., Patrick, S., Starkey, D. E., Gilbert, J., Geiser, D. M. and Nowicki, T. W. 2008. An adaptive evolutionary shift in Fusarium head blight pathogen populations is driving the rapid spread of more toxigenic Fusarium graminearum in North America. Fungal Genet. Biol. 45:473-484.

Zhang, H., Zhang, Z., van der Lee, T., Chen, W. Q., Xu, J., Xu, J. S., Yang, L., Yu, D., Waalwijk, C. and Feng, J. 2010. Population genetic analyses of Fusarium asiaticum populations from barley suggest a recent shift favoring $3 \mathrm{ADON}$ producers in southern China. Phytopathology 100:328-336.

Zhang, J. B., Wang, J. H., Gong, A. D., Chen, F. F., Song, B., Li, X., Li, H. P., Peng, C. H. and Liao, Y. C. 2013. Natural occurrence of Fusarium head blight, mycotoxins and mycotoxinproducing isolates of Fusarium in commercial fields of wheat in Hubei. Plant Pathol. 62:92-102. 